\title{
Adaptive Focused Ion Beam Milling through Machine Learning Algorithm Integration
}

Max Turnquist, Peter Lewis, Troy Lau, Elizabeth Brundage and Andrew Magyar

Draper, United States

Focused Ion Beam (FIB) milling is used to create features ranging from 10s of nanometers to hundreds of microns. However, milling accuracy with respect to design is dependent on substrate materials, mill features/parameters, and sputtering. FIB milling requires a significant amount of subject-matter-expertise, and standard milling recipes fail to account for most material subsets, changing mill rates of the sample and sputtering of the milled material in and around the target area. The goal of this work is to develop an adaptive milling machine learning algorithm in order to accurately capture and improve upon this knowledge, enabling automated and efficient milling features into substrates in the FIB. The adaptive milling system is designed to facilitate FIB milling through the abstraction of individual commands and controls to a process of supplying a desired model, and letting a machine learning model render it. The auto-corrective milling and deposition system is designed to work iteratively, therefore there is no true start or end to the process, there are artificial tolerance bright-lines which determine its function. The core iteration loop is comprised of four phases: collection, reconstruction, recognition, and execution. In the collection phase, a series of detectors inside the FIB collect electron micrographs of the target from different angles. These angles are crucial and provide multiple perspectives for reconstruction. In the second phase, reconstruction, images are overlaid, rectified, and processed with photogrammetry techniques to create a 3D point cloud of the actual 3D object. This point cloud is then compared against the desired model, and differences are extracted to their own point-cloud. In the recognition phase, the difference point cloud is fitted with a variety of primitives- each primitive corresponding to its own set of pre-established FIB commands. The best fitting primitive is then reduced to execution code, which is sent to the FIB. The FIB executes the instructions as best it can- this is where the structure of the auto-correction becomes useful. FIB milling is not always a straightforward process- often there is non-homogenous milling and sputtering. To our adaptive milling system, these errors are no different than the substrate, and with the new cycle, the reconstruction identifies and removes them as if they were any other topology. This work focuses on a foundational application of the algorithm; milling letters into a homogenous silicon substrate. The path forward to applying it to more complex (e.g. heterogeneous substrates, arbitrary mill shapes) applications will be discussed. The FIB algorithm will leverage its ability to identify and correct milling deformities, on a scale not possible by a human user, while also performing non-intuitive actions to complete the task. This work will serve as the foundation for complex material system milling. 